\title{
Suppression of Drift in FET-type Gas Sensor Having WS 2 Nanoparticles Using Pulse Measurement
}

\author{
Yujeong Jeong ${ }^{1}$, Jongmin Shin ${ }^{1}$, Yoonki Hong ${ }^{1}$, Meile Wu ${ }^{1}$, Seongbin Hong ${ }^{1}$, Dongkyu Jang ${ }^{1}$, Ki Chang \\ Kwon ${ }^{2}$, Seokhoon Choi ${ }^{2}$. Ho Won Jang ${ }^{2}$, and Jong-Ho Lee ${ }^{1}$ \\ 1 Department of Electrical Eng., Seoul National University, Seoul 08826, Republic of Korea \\ 2 Department of Materials Science and Eng., Research Institute of Advanced Materials, Seoul National \\ University, Seoul 08826, Republic of Korea \\ jhl@snu.ac.kr
}

\begin{abstract}
:
In this paper, sensing characteristics of the FET-type gas sensor having $\mathrm{WS}_{2}$ nanoparticles as a sensing layer deposited using ink-jet printer are analyzed with various measurement conditions. In case of using DC measurement method, the transfer curve has a large hysteresis gap due to charge trapping in the sensing layer. For this reason, we adopt a pulse measurement. Transient response of the FET-type gas sensor is measured with $\mathrm{NO}_{2}$ concentrations using $\mathrm{DC}$ and pulse measurement methods. Through adopting pulse measurement, drift characteristics of the gas sensor are suppressed and recovery characteristics are improved. As $\mathrm{NO}_{2}$ concentration increases, response rate of the gas sensor increases. As a result, the gas sensing characteristics of the FET-type sensor having $\mathrm{WS}_{2}$ sensing layer are improved effectively by using pulse measurement method.
\end{abstract}

Key words: TMDCs, $\mathrm{WS}_{2}, \mathrm{NO}_{2}$ sensor, MOSFET, pulse measurement

\section{Experimental Preparation}

Fig. 1. (a) is a microscopic image of the fabricated field effect transistor(FET)-type gas sensor having horizontally formed floating-gate (FG). The fabrication process of the gas sensor has been reported in our previous work [1]. Before forming the sensing layer, SU-8 patterning is performed to protect metal electrodes since metal electrodes can be damaged by ink solutions. In final steps, $\mathrm{WS}_{2}$ nanoparticles as a sensing layer is printed using ink-jet printer using $W_{2}$ solution. The solution having $W S_{2}$ nanoparticles is produced through exfoliation method from $\mathrm{WS}_{2}$ micro powder mixed in solvent. Fig. 1. (b) shows that a SEM image of ink-jet printed $W_{2}$ nanoparticles on a sensing area. After $W_{2}$ sensing layer is printed, $\mathrm{H}_{2}$ annealing is performed at $300^{\circ} \mathrm{C}$ to remove surfactant of $\mathrm{WS}_{2}$ solution.

\section{Experimental Results}

The characteristics of the synthesized $\mathrm{WS}_{2}$ nanoparticles are measured by XPS spectrum analysis. As shown in Fig. 2, which is XPS spectrum of the synthesized $\mathrm{WS}_{2}$ nanoparticles, $1 \mathrm{~T}$-phase $\mathrm{WS}_{2}$ nanoparticles are well- synthesized. Fig. 3 shows that hysteresis gap of transfer curve $\left(I_{D}-V_{C G}\right)$ is a relatively large when the curve is measured using DC measurement. A hysteresis gap of the FET-type gas sensor with a $W_{2}$ sensing layer is attributed to the charge trapping in the $\mathrm{WS}_{2}$ nanoparticles and the interface between the $W_{S_{2}}$ sensing layer and the $\mathrm{O} / \mathrm{N} / \mathrm{O}$ stack [2]. Especially in case of DC measurement, more charges are trapped since a constant bias is applied to the controlgate (CG) of the gas sensor for a long time. Fig. 4 shows that time response of the gas sensor with different $\mathrm{NO}_{2}$ concentrations ranging from $2.5 \mathrm{ppm}$ to $10 \mathrm{ppm}$ using DC measurement at $100{ }^{\circ} \mathrm{C}$. As shown in Fig. 4, the recovery characteristics of the gas sensor are degraded by the drift effect originated from the charge trapping. To solve this problem, we adopt a pulse measurement. The pulse measurement is carried out using the pulse scheme in the inset of Fig. 3, which has an on-time of $200 \mu \mathrm{s}$, offtime of $0.01 \mathrm{~s}$. In the pulse measurement, control gate is biased for a short on-time, and biased to OV for off-time. Therefore, much less charge is trapped, and then the drift of the gas sensor is suppressed. Fig. 5 shows that $\mathrm{NO}_{2}$ response of the gas sensor with different $\mathrm{NO}_{2}$ 
concentrations using pulse measurement at $100^{\circ} \mathrm{C}$. Contrary to transient curves using DC measurement method, drift characteristics of the gas sensor is suppressed by using a $V_{C G}$ pulse of $-0.9 \mathrm{~V}$. Thus, as shown in Fig. 5, gas response and recovery characteristics are improved.

\section{References}

[1] Chang-Hee Kim., et al. A New Gas Sensor Based on MOSFET Having a Horizontal Floating-Gate, IEEE ELECTRON DEVICE LETTERS, 35.2 (2014); doi: 10.1109/LED.2013.2294722

[2] Sung Tae Lee., et al. Accurate extraction of WSe2 FETs parameters by using pulsed I-V method at various temperatures, Nano convergence, 3.1 (2016); doi: 10.1186/s40580016-0091-9

(a)

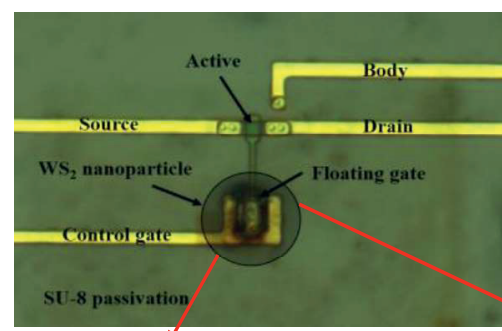

(b)

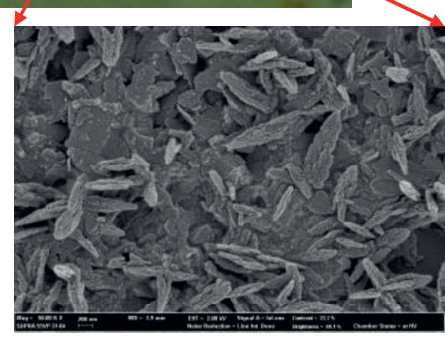

Fig.1. (a) The optical top view of FET-type gas sensor having horizontal floating-gate and inkjet printed $W S_{2}$ sensing layer. (b) A SEM image of Ink-jet printed $W S_{2}$ nanoparticles.
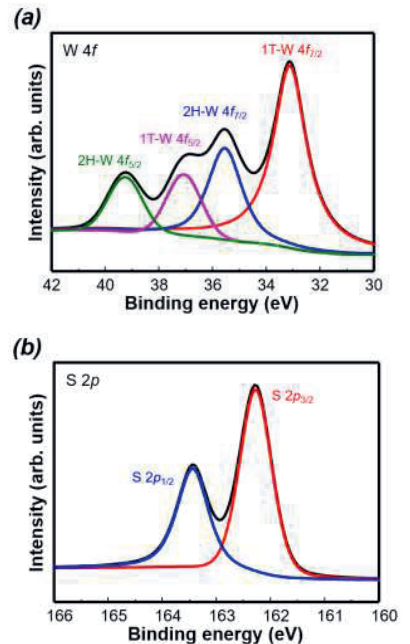

Fig.2. XPS spectrum of the synthesized $W S_{2}$ nanoparticles.: (a) W $4 f$ region, (b) $S 2 p$ region.

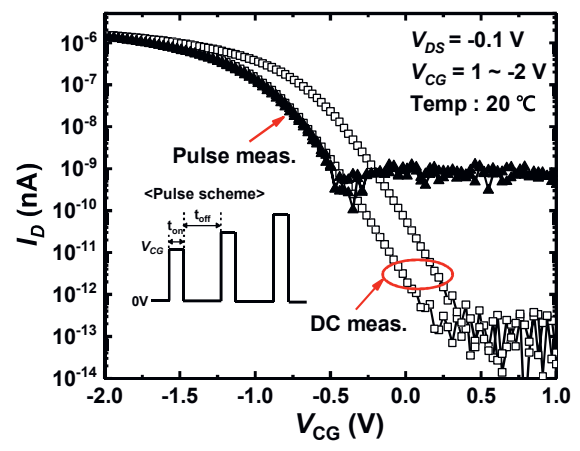

Fig.3. Transfer characteristics $\left(I_{D}-V_{C G}\right)$ of the $W S_{2}$ gas sensor at $20^{\circ} \mathrm{C}$ using DC measurement and Pulse measurement. The pulse scheme in the inset is used for pulse measurement.

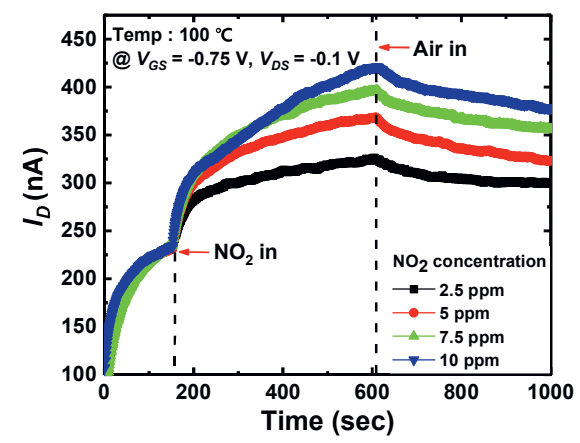

Fig.4. Transient response of FET-type gas sensor having $W_{2} S_{2}$ nanoparticles using $D C$ measurement at $100{ }^{\circ} \mathrm{C}$ as a parameter of $\mathrm{NO}_{2}$ concentration from 2.5 to $10 \mathrm{ppm}$.

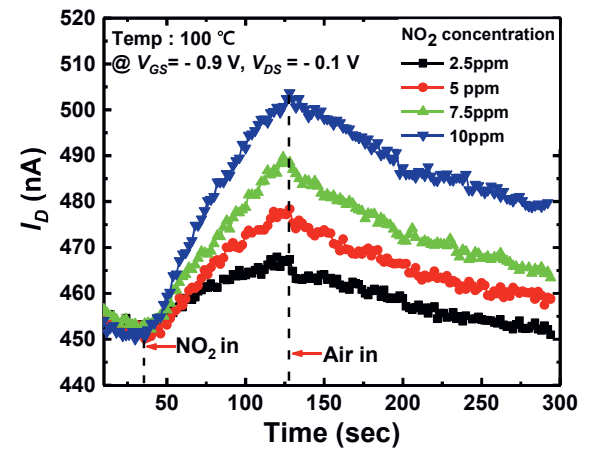

Fig.5. Transient response of FET-type gas sensor having $W_{2}$ nanoparticles using pulse measurement at $100^{\circ} \mathrm{C}$ as a parameter of $\mathrm{NO}_{2}$ concentration from 2.5 to $10 \mathrm{ppm}$.

Acknowledgements: This work was supported by the National Research Foundation of Korea (NRF2016R1A2B3009361) and the Brain Korea 21 Plus Project in 2018. 